\title{
Neurorehabilitation of social dysfunctions: a model-based neurofeedback approach for low and high-functioning autism
}

\author{
Jaime A. Pineda *, Elisabeth V. C. Friedrich and Kristen LaMarca \\ Department of Cognitive Science, University of California, San Diego, La Jolla, CA, USA
}

\author{
Edited by: \\ Aleksandra Vuckovic, University of \\ Glasgow, UK \\ Reviewed by: \\ Frank Pollick, University of Glasgow, \\ UK \\ Lucia Billeci, National Research \\ Council of Italy, Italy \\ ${ }^{*}$ Correspondence: \\ Jaime A. Pineda, Department of \\ Cognitive Science, University of \\ California, 9500 Gilman Drive, San \\ Diego, La Jolla, CA 92093, USA \\ e-mail:pineda@ \\ cogsci.ucsd.edu
}

\begin{abstract}
Autism Spectrum Disorder (ASD) is an increasingly prevalent condition with core deficits in the social domain. Understanding its neuroetiology is critical to providing insights into the relationship between neuroanatomy, physiology and social behaviors, including imitation learning, language, empathy, theory of mind, and even self-awareness. Equally important is the need to find ways to arrest its increasing prevalence and to ameliorate its symptoms. In this review, we highlight neurofeedback studies as viable treatment options for highfunctioning as well as low-functioning children with ASD. Lower-functioning groups have the greatest need for diagnosis and treatment, the greatest barrier to communication, and may experience the greatest benefit if a treatment can improve function or prevent progression of the disorder at an early stage. Therefore, we focus on neurofeedback interventions combined with other kinds of behavioral conditioning to induce neuroplastic changes that can address the full spectrum of the autism phenotype.
\end{abstract}

Keywords: neurofeedback training, autism, low functioning autism, autism spectrum disorders, intervention studies, classical conditioning

\section{AIM}

In this review, we highlight preliminary yet promising observations to support the hypothesis that neurofeedback training (NFT) in combination with a new behavioral intervention, TAGteach, is a viable treatment option not only for highfunctioning but also for low-functioning children with ASD.

\section{SYMPTOMS}

Autism Spectrum Disorder (ASD) is now estimated to affect 1 in 68 children (Baio, 2014), who show marked deficits in social and communicative skills, including imitation, empathy, and shared attention, as well as restricted interests and repetitive patterns of behaviors. These problems significantly affect social interactions and prevent children from establishing normal social relationships with others. The ASD phenotype varies in severity and character, encompassing individuals who are asocial but otherwise high-functioning (sometimes at the "savant" level), and low-functioning non-verbal individuals. The distinction between low and high-functioning autism is typically based on the child's IQ, with the cutoff being around 80. At the high-functioning end of the spectrum, the deficits primarily impair social interactions and prevent children from establishing adequate relations with others. At the low-functioning end, children show interactions mainly with the goal of behavior regulation such as protesting rather than social engagement (Maljaars et al., 2012).

\section{CAUSES AND BRAIN CORRELATES}

Different models to explain autism have been proposed, but the neuroetiology of autism is not yet entirely understood.
Rubenstein and Merzenich (2003) have proposed an "increased ratio of excitation/inhibition in sensory, mnemonic, social and emotional systems", which can be caused by a combination of environmental and genetic factors. Another working hypothesis is that a dysfunction in motor processing, specifically in the mirror neuron system (MNS; di Pellegrino et al., 1992; Williams et al., 2001; Rizzolatti and Craighero, 2004), is an underlying factor in the condition. In one anatomical MRI study, Hadjikhani et al. (2006) examined 14 high-functioning adults with ASD and observed significant cortical thinning compared to matched control participants. These differences were found in areas considered part of the classic MNS, such as the inferior frontal gyrus (IFG), bilateral inferior parietal lobule (IPL), as well as right superior temporal sulcus (STS). These findings have been strengthened by significant correlations with autism symptoms, as diagnosed by the autism diagnostic interview-revised or ADI-R (Lord et al., 1994). Williams et al. (2006) studied 16 adolescents with ASD during finger movement imitation. Although no effects were detected in IFG, a comparison with matched typically developing (TD) children showed reduced activation for the ASD group in bilateral IPL. Dapretto et al. (2006) reported reduced activation in IFG in another fMRI study in which they tested nine boys with ASD during imitation of emotional facial expressions. Although children were able to perform the imitation task, significantly reduced activation in IFG was detected bilaterally in a comparison with controls. In contrast, Martineau et al. (2010) reported hyperactivation of the pars opercularis (belonging to the MNS) during observation of human motion in autistic subjects compared to controls. Baastiaansen et al. 
(2011) reported that IFG activity during the observation of facial expressions increased with age in subjects with autism, but not in controls, suggesting improved social functioning with age. In terms of functional connectivity, Villalobos et al. (2005) found reduced fMRI connectivity between primary visual cortex and bilateral IFG during visuomotor coordination in eight participants with ASD, compared to matched TD participants. Likewise, studies using resting state (rs)-magnetoencephalography (MEG) or quantitative electroencephalography (QEEG) support the notion of dysfunctional connectivity in ASD. Tsiaras et al. (2011) showed reduced interdependence strength within bilateral frontal and temporal sensors, as well as between temporal sensors and other recording sites in a group of ASD participants. Cornew et al. (2012) indicated that children with ASD exhibited regionally specific elevations in delta $(1-4 \mathrm{~Hz})$, theta (4-8 Hz), alpha $(8-12 \mathrm{~Hz})$, and high frequency $(20-120 \mathrm{~Hz})$ power, supporting an imbalance of neural excitation/inhibition as a neurobiological feature of ASD. Billeci et al. (2013) showed that children with ASD present several differences in power spectra, coherence, and symmetry measures compared to controls. This is true both when the signals are acquired in resting conditionswith either open or closed eyes_-and when specific tasks are performed. For all these reasons it is speculated that abnormal functional connections exist that can lead to ineffective or atypical neural communication, which in turn may impede early affective, social and communicative development. This suggests that a reasonable therapeutic approach for the treatment of ASD is to normalize abnormal functional connections (Pineda et al., 2012). However, most of these findings rely on data from highfunctioning individuals (Dapretto et al., 2006). As IQ correlates with brain volume (Posthuma et al., 2002; Posthuma and Polderman, 2013), brain structure (Price et al., 2013) and brain function (van den Bos et al., 2012), it has yet to be shown if a generalization of these results to the lower end of the spectrum is justified.

\section{TREATMENTS}

In clinical studies, the most effective type of therapy for ASD has been behavioral intervention, with an efficacy rate of approximately 48\% (Lovaas, 1987; McEachin et al., 1993; Smith et al., 2009). However, behavioral therapy is time consuming and costly for such a low potential benefit, making it entirely out of reach to the majority of the affected population, especially in developing countries. Moreover, research aimed at interventions is predominantly conducted with highfunctioning adults or adolescents and a review of the literature confirms that social skills training for ASD mostly involves high-functioning children (Cappadocia et al., 2012; Wainer and Ingersoll, 2013). However, it is the younger lower-functioning groups that have the greatest need for diagnosis and treatment, who have the greatest barrier to communication (and hence the greatest need for replacement or enhancement of communication), and who may experience the greatest benefit if such tools can improve function or prevent progression of the disorder at an early stage. Ben et al. $(2008,2011)$ reported better outcome in behavioral therapy for children with an IQ above 70 and less severe symptoms than for children with an IQ below
70 and more deficits. This emphasizes that different treatment approaches are needed and should be used for lower functioning children on the spectrum. Thus, alternative interventions that normalize social behavior would be beneficial and warrant serious consideration.

One alternative to behavioral therapy is NFT. NFT allows for visualization of brain activity to be fed back to a user by means of a computer in a closed "neurofeedback" loop, allowing subjects to learn to control the natural operation of brain rhythms in vivo and in near real time (Nowlis and Kamiya, 1970; Delorme and Makeig, 2004; Delorme et al., 2011). Brain electrical rhythms are instantiated across different spatial scales (Buzsáki and Draguhn, 2004) from single neurons (Hutcheon and Yarom, 2000), to neuronal circuits (Whittington et al., 1997), to re-entrant thalamocortical and large-scale cortico-cortical networks (Lorincz et al., 2009). It is assumed that these rhythms enable the dynamic routing and gating of information via the synchronization of various bits of information (Salinas and Sejnowski, 2001; Schoffelen et al., 2005; Jensen and Mazaheri, 2010). From a systems level perspective, electroencephalographic (EEG) responses to sensory stimuli can partially be explained by transient, stimulus-induced adjustment in the phase of ongoing rhythms via phase-resetting (Sayers et al., 1974; Makeig et al., 2002; Klimesch et al., 2007). The possibility of self-directed modulation of these rhythms and phase adjustments raises an interesting set of questions. Is it possible to promote/enhance or inhibit/suppress rhythmic oscillations in distinct neural networks in vivo? Can we modulate these rhythms volitionally through some periodic internal input or drive? Theoretically, a functional impact is possible through the modulation of brain rhythms that may play a causal role in specific cognitive functioning.

There is consensus that EEG activity recorded on the scalp arises mainly from cortex (Pantev et al., 1991; Llinás and Ribary, 1993). Neurons firing in synchrony while an individual is at rest produce large amplitude oscillations detected over various brain regions (Hari et al., 1997; Klimesch, 1997; Pfurtscheller et al., 1997, 2000; Muthukumaraswamy and Johnson, 2004; Muthukumaraswamy et al., 2004). The mu rhythm is such an oscillation in the $8-13 \mathrm{~Hz}$ band, limited to brief periods of 0.5-2 s duration and recorded in the absence of movement over sensorimotor cortex. Activation by self-movement, the observation of movement, and even the imagination of movement produces desynchronization and a suppression of $\mathrm{mu}$ rhythm activity (Salmelin and Hari, 1994; Pfurtscheller et al., 1997). The link between mu rhythms and mirroring activity was first proposed by Altschuler et al. (1998), and thereafter by other researchers (Cochin et al., 1998, 1999; Hari et al., 2000). More recent studies have found that mu rhythm is modulated by objectdirected actions (Muthukumaraswamy and Johnson, 2004). Since it is generated by activity in sensorimotor areas and mirror neurons have been located primarily in premotor areas, it is hypothesized that mu rhythm indexes downstream modulation of primary sensorimotor areas by mirroring activity in frontal cortex (Muthukumaraswamy and Johnson, 2004; Muthukumaraswamy et al., 2004; Oberman et al., 2005; Pineda, 2005). Furthermore, TD individuals can learn to modulate mu rhythms via NFT (Pineda et al., 2000, 2003), and while normal individuals exhibit 
mu suppression to both self-directed and observed movement, high-functioning ASD individuals fail to exhibit mu suppression to observed movement (Oberman et al., 2005).

Most NFT approaches use a simple visual stimulus or game to train individuals to increase/decrease a particular bandwidth of the EEG signal. With training, the majority of individuals develop a high level of conscious and unconscious control over their brain activity. During training, subjects are exposed to the same visual/auditory feedback or reward stimuli, and hence the entrained EEG differences most likely represent the modulation of some internal brain state associated with the event rather than to external factors. While the precise mechanisms of how using neurofeedback can induce changes in the brain are unclear, the evidence suggests they capitalize on the innate plasticity of the brain to produce neural, functional, and ultimately behavioral changes. Furthermore, the use of QEEG (Cantor and Chabot, 2009; Coben and Myers, 2010; Thompson et al., 2010a,b) combined with specific and individualized protocols (e.g., amplitude and coherence training) can help fit the training to the heterogeneity of autistic symptomatology.

The majority of studies using neurofeedback for ASD includes children at the higher end of the autism spectrum and these studies have reported significant normalization of brain functioning as well as improvement in behavior and cognitive function (Pineda et al., 2008; Kouijzer et al., 2009; Coben et al., 2010). However, the generalization of these findings to adults or very young and lower functioning children is not yet well examined (Coben et al., 2010). One concern is whether nonverbal children even understand instructions and can process the meaning of feedback. High functioning individuals seem to process external, concrete feedback in a way similar to their TD peers (Larson et al., 2012). However, a recent study suggested that nonverbal low functioning individuals with ASD are able to attribute goals to other persons but are not able to consider the person's circumstances or the context to interpret the actions and attribute intentions as most typical developing children do (Somogyi et al., 2013). Indeed, neurofeedback-based learning is correlated with IQ (van den Bos et al., 2012).

\section{TREATMENT FOR LOW-FUNCTIONING CHILDREN WITH} AUTISM: TEACHING WITH ACOUSTICAL GUIDANCE AND NFT Operant and classical conditioning principles have been key to the behavioral treatment of autism for decades (Helm, 1976; Neuringer, 2002; Pickett et al., 2009). Secondary or conditioned reinforcers, such as auditory markers, have been shown to enhance learning, including the learning of complex sequences of behaviors, through the value acquired from their associations with primary reinforcers. As lower functioning children with ASD have a greater degree of social, language, and other behavioral deficits, they have less available treatment options and tend to be precluded from research. An auditory marker appears well suited for the enhancement of skill-teaching in this population in that it eliminates the social features of verbal praise and is more precise than verbal reinforcement. Teaching with Acoustic Guidance or TAGteach, ${ }^{1}$ a new behavioral intervention that uses

\footnotetext{
${ }^{1}$ http://www.TAGteach.com
}

a conditioned auditory reinforcer to mark and shape behaviors in successive approximations, was recently used to accelerate learning in a child with ASD who had difficulty learning through a traditional behavioral approach (Persicke et al., 2014). Case reports on the TAGteach webpage ${ }^{2}$ and several case presentations at the Applied Behavioral Analysis International (ABAI) meeting have also reported that TAGteach can enhance skill acquisition rates in children with ASD who have more difficulty learning through traditional means.

Ueda (2007) demonstrated that TAGteach was effective in easing the difficulties with transitions between tasks experienced by a 3 year old with autism. After implementing TAG methodology to increase compliance with transitions, the amount of time and number of prompts decreased compared to baseline data. In a second case study of an 8 year old female diagnosed with autism, Ueda showed that TAG decreased the number of prompts and increased the level of independence in fine motor imitation skills and requesting behaviors. Also Hanson and Madden (2007) demonstrated an increase in acquisition rates for the mastery of several different target behaviors in 5 case studies with the use of TAGteach compared to the traditional operant strategies that were used in collecting baseline data. Rosenblum (2007) used TAG to teach typing to a 9-year-old male student with autism. The subject learned to type words via TAGteach with $62.3 \%$ fewer prompts than words for which TAGteach was not used. Also, Gutierrez (2007) investigated the effects of TAG on acquisition rates of imitation behaviors in two male children with autism, ages 2 and 3 , following little or no progress during baseline conditions that used traditional discrete trial methods. Following 7 months of baseline data with no progress, the 3-year-old student showed $90 \%$ accuracy for two imitation responses in just 15 sessions once TAG was used. The 2-year-old student was able to acquire target behaviors in less than four TAGteach sessions following a baseline of eight sessions without TAG that showed minimal acquisition. Winkle (2007) used TAGteach with a 12 year old male diagnosed with autism to teach a social interaction by approaching a peer, getting the peer's attention, making eye contact, and saying hello. She showed that the time for the participant to perform a behavior and the average number of cues per interaction decreased while the average number of eye contact occurrences per session increased when using TAGteach. Winkle also noted improvement in the participant's affect and communication skills. Though preliminary, the aforementioned case studies, highlight the potential of TAGteach to enhance shaping procedures in children with ASD that have difficulty learning behaviors through traditional behavioral methods could prove to be highly valuable to the field.

There is a need for the empirical exploration of novel treatments that target the core deficits of autism, align themselves with prominent neuroetiological theories, and are suitable for lower functioning populations. To that end, we have combined TAGteach with NFT to bridge the gap that currently exists and completed a case series of seven children with autism and with impairments that would typically preclude them from research participation in the typical study with high functioning children.

\footnotetext{
${ }^{2}$ http://www.TAGteach.com/Autism_And_Special_Education
} 
The study aimed to investigate (a) whether TAGteach could be used to behaviorally prepare children to perform the skills required of an NFT intervention and EEG outcome tasks; and (b) whether electrophysiological changes or improvements in behavioral outcomes would occur in participants learning to self-regulate mu rhythms through operant strategies.

Participants were required to have a diagnosis of Autistic Disorder, be between the ages of 8 and 12, and have an IQ below 80. TAGteach was first used to shape a set of prerequisite behaviors in all participants so they could undergo an electrophysiological assessment, namely the Mu Suppression Index (MSI) and NFT thereafter. $\mathrm{Mu}$ suppression indices were calculated as the ratio of power during biological, goal-directed, and social action conditions relative to the power during the non-biological action condition, which is a type of movement that does not typically produce mu rhythm suppression. Following completion of pretest measures, participants attended NFT sessions for 30-45 min 2 or 3 times per week, totaling $20 \mathrm{~h}$ over 20 weeks. They were trained to control power in the mu band $(8-13 \mathrm{~Hz}$ over electrode site $\mathrm{C} 4)$ as well as a second frequency range $(43-59 \mathrm{~Hz})$ recorded over $\mathrm{C} 4$ that reflects muscle movement artifact. A secondary auditory reinforcer, used in the TAGteach part of the study, continued to mark when participants exceeded the mu power threshold during NFT. This was followed by the primary reinforcer, which included either a videogame or preferred DVD movie that played upon mu activity exceeding a threshold. Thresholds were set based on initial individual assessment and increased when participants showed learning. Following the completion of treatment, post assessments were completed by participant and caregiver.

The results were highly encouraging in that all participants learned to perform the behaviors required of NFT and EEG outcome tasks in an average of $5 \mathrm{~h}$ over 6 TAGteach sessions (see Figure 1). The administration of NFT was entirely feasible via TAGteach in these children, providing a promis-

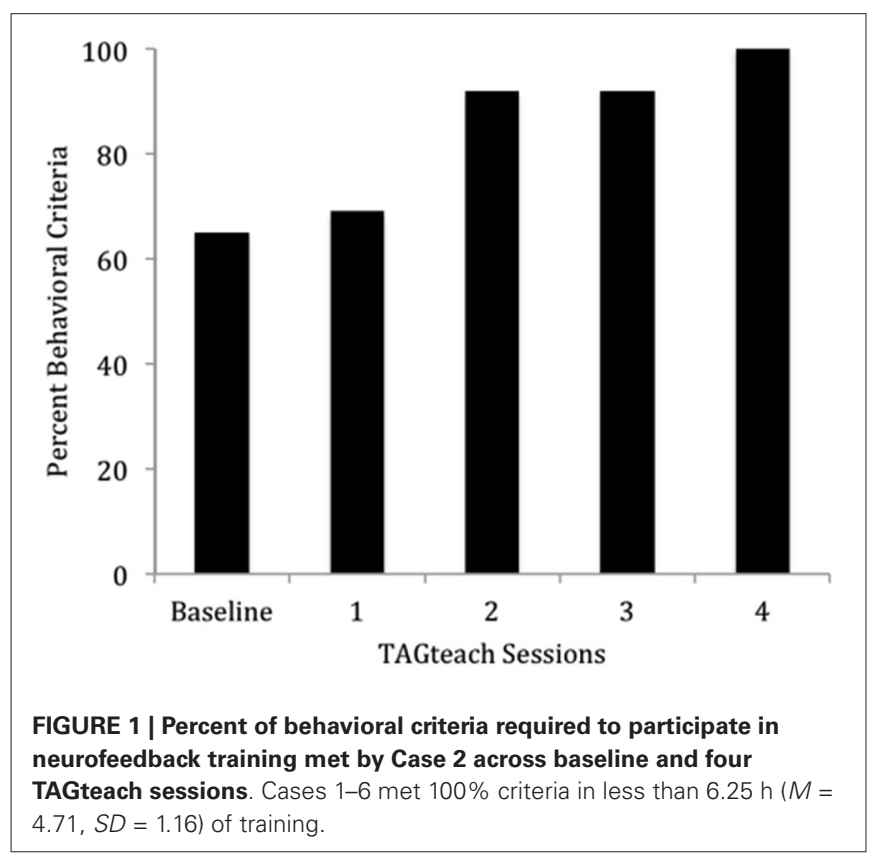

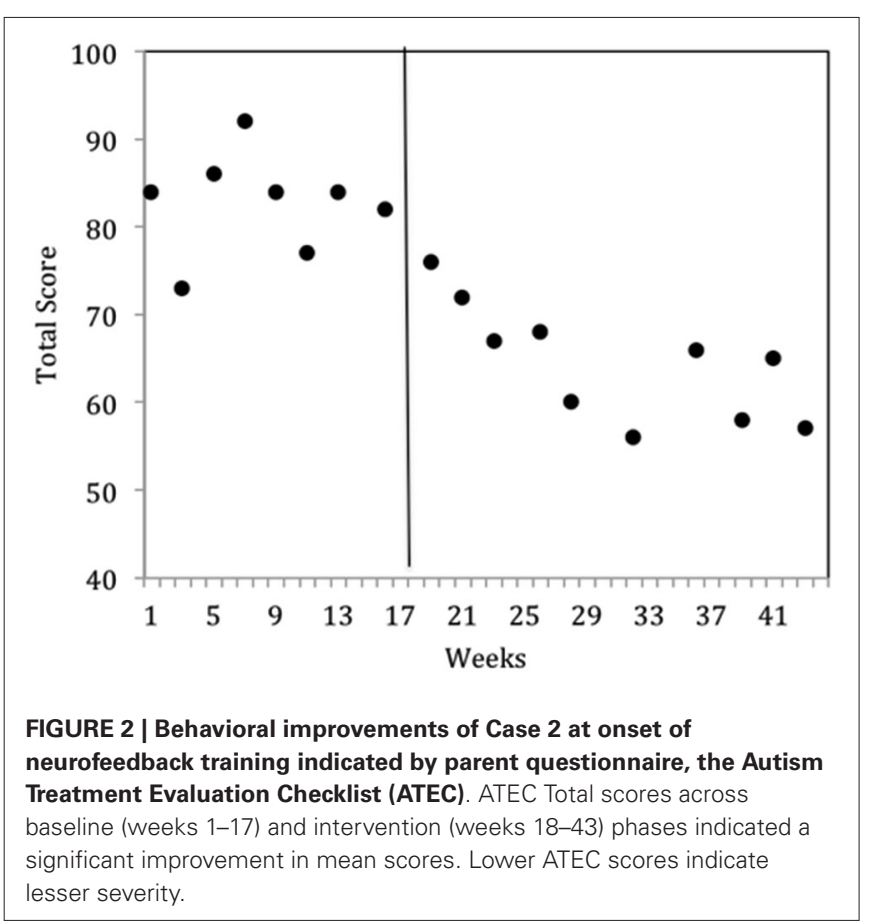

ing way to normalize mu suppression responses and improve behavior (see Figure 2). Furthermore, the results suggested that biofeedback-assisted TAGteach training could indeed help children with low functioning autism to better sustain complex skills and reduce artifact-creating behaviors for the required durations. These findings support TAGteach as a feasible method of preparing lower functioning children on the spectrum to cooperate with research tasks and participate in an otherwise inaccessible, or difficult to implement, treatment.

\section{CONCLUSION}

There are few research studies including children on the lower end of the autism spectrum, which creates poor treatment options for this population. In this review we present TAGteach in combination with NFT as a promising alternative that may be suitable for low functioning children on the spectrum.

\section{REFERENCES}

Altschuler, E. L., Vankov, A., Wang, V., Ramachandran, V. S., and Pineda, J. A. (1998). Person see, person do: human cortical electrophysiological correlates of monkey see monkey do cells. J. Cogn. Neurosci. Suppl. S:91, Abstract.

Baastiaansen, J. A., Thioux, M., Nanetti, L., van der Gaag, C., Ketelaars, C., Minderaa, R., et al. (2011). Age-related increase in inferior frontal gyrus activity and social functioning in autism spectrum disorder. Biol. Psychiatry 69, 832838. doi: 10.1016/j.biopsych.2010.11.007

Baio, J. (2014). Prevalence of autism spectrum disorder among children aged 8 years-autism and developmental disabilities monitoring network, 11 sites, United States, 2010. MMWR Surveill. Summ. 63, 1-21.

Ben, I. E., Lahat, E., and Zachor, D. A. (2011). Advanced parental ages and low birth weight in autism spectrum disorders-rates and effect on functioning. Res. Dev. Disabil. 32, 1776-1781. doi: 10.1016/j.ridd.2011.03.004

Ben, I. E., Lahat, E., Burgin, R., and Zachor, A. D. (2008). Cognitive, behavior and intervention outcome in young children with autism. Res. Dev. Disabil. 29, 447458. doi: 10.1016/j.ridd.2007.08.003 
Billeci, L., Sicca, F., Maharatna, K., Apicella, F., Narzisi, A., Campatelli, G., et al. (2013). On the application of quantitative EEG for characterizing autistic brain: a systematic review. Front. Hum. Neurosci. 7:442. doi: 10.3389/fnhum.2013. 00442

Buzsáki, G., and Draguhn, A. (2004). Neuronal oscillations in cortical networks. Science 304, 1926-1929. doi: 10.1126/science.1099745

Cantor, D. S., and Chabot, R. (2009). QEEG studies in the assessment and treatment of childhood disorders. Clin. EEG Neurosci. 40, 113-121. doi: 10. 1177/155005940904000209

Cappadocia, M. C., Weiss, J. A., and Pepler, D. (2012). Bullying experiences among children and youth with autism spectrum disorders. J. Autism Dev. Disord. 42, 266-277. doi: 10.1007/s10803-011-1241-x

Coben, R., and Myers, T. E. (2010). The relative efficacy of connectivity guided and symptom based EEG biofeedback for autistic disorders. Appl. Psychophysiol. Biofeedback 35, 13-23. doi: 10.1007/s10484-009-9102-5

Coben, R., Linden, M., and Myers, T. E. (2010). Neurofeedback for autistic spectrum disorder: a review of the literature. Appl. Psychophysiol. Biofeedback 35, 83-105. doi: 10.1007/s10484-009-9117-y

Cochin, S., Barthelemy, C., Lejeune, B., Roux, S., and Martineau, J. (1998). Perception of motion and qEEG activity in human adults. Electroencephalogr. Clin. Neurophysiol. 107, 287-295. doi: 10.1016/s0013-4694(98) 00071-6

Cochin, S., Barthelemy, C., Roux, S., and Martineau, J. (1999). Observation and execution of movement: similarities demonstrated by quantified electroencephalography. Eur. J. Neurosci. 11, 1839-1842. doi: 10.1046/j.1460-9568.1999. 00598.x

Cornew, L., Roberts, T. P., Blaskey, L., and Edgar, J. C. (2012). Resting-state oscillatory activity in autism spectrum disorders. J. Autism Dev. Disord. 42, 1884-1894. doi: 10.1007/s10803-011-1431-6

Dapretto, M., Davies, M. S., Pfeifer, J. H., Scott, A. A., Sigman, M., Bookheimer, S. Y., et al. (2006). Understanding emotions in others: mirror neuron dysfunction in children with autism spectrum disorders. Nat. Neurosci. 9, 28-30. doi: 10. 1038/nn1611

Delorme, A., and Makeig, S. (2004). EEGLAB: an open source toolbox for analysis of single-trial EEG dynamics including independent component analysis. J. Neurosci. Methods 134, 9-21. doi: 10.1016/j.jneumeth.2003. 10.009

Delorme, A., Mullen, T., Kothe, C., Akalin, A. Z., Bigdely-Shamlo, N., Vankov, A., et al. (2011). EEGLAB, SIFT, NFT, BCILAB and ERICA: new tools for advanced EEG processing. Comput. Intell. Neurosci. 2011:130714. doi: 10. $1155 / 2011 / 130714$

di Pellegrino, G., Fadiga, L., Fogassi, L., Gallese, V., and Rizzolatti, G. (1992). Understanding motor events: a neurophysiological study. Exp. Brain Res. 91, 176-180. doi: 10.1007/bf00230027

Gutierrez, M. R. (2007). "Implementing TAG to increase preacademic skills in the school environment," in Teaching with Acoustical Guidance (TAG) at Applied Behavior Consultants Inc. Symposium Conducted at the 25th Annual Western Regional Conference, eds J. Morrow and J. Vargas (Burlingame, CA: California Association for Behavior Analysis).

Hadjikhani, N., Joseph, R. M., Snyder, J., and Tager-Flusberg, H. (2006). Anatomical differences in the mirror neuron system and social cognition network in autism. Cereb. Cortex 16, 1276-1282. doi: 10.1093/cercor/bhj069

Hanson, R., and Madden, K. (2007). "Implementing TAG to increase self-help skills in the home environment," in Teaching with Acoustical Guidance (TAG) at Applied Behavior Consultants Inc. Symposium Conducted at the 25th Annual Western Regional Conference, eds J. Morrow and J. Vargas (Burlingame, CA: California Association for Behavior Analysis).

Hari, R., Levanen, S., and Raij, T. (2000). Timing of human cortical functions during cognition: role of MEG. Trends Cogn. Sci. 4, 455-462. doi: 10.1016/s13646613(00)01549-7

Hari, R., Salmelin, R., Makela, J. P., Salenius, S., and Helle, M. (1997). Magnetoencephalographic cortical rhythms. Int. J. Psychophysiol. 26, 51-62. doi: 10. 1016/s0167-8760(97)00755-1

Helm, D. (1976). Psychodynamic and behavior modification approaches to the treatment of infantile autism empirical similarities. J. Autism Child Schizophr. 6, 27-41. doi: 10.1007/bf01537940

Hutcheon, B., and Yarom, Y. (2000). Resonance, oscillation and the intrinsic frequency preferences of neurons. Trends Neurosci. 23, 216-222. doi: 10. 1016/s0166-2236(00)01547-2
Jensen, O., and Mazaheri, A. (2010). Shaping functional architecture by oscillatory alpha activity: gating by inhibition. Front. Hum. Neurosci. 4:186. doi: 10.3410/f. 6253958.6325058

Klimesch, W. (1997). EEG-alpha rhythms and memory processes. Int. J. Psychophysiol. 26, 319-340. doi: 10.1016/s0167-8760(97)00773-3

Klimesch, W., Sauseng, P., Hanslmayr, S., Gruber, W., and Freunberger, R. (2007). Event-related phase reorganization may explain evoked neural dynamics. $\mathrm{Neu}$ rosci. Biobehav. Rev. 31, 1003-1016. doi: 10.1016/j.neubiorev.2007.03.005

Kouijzer, M. E. J., de Moor, J. M. H., Gerrits, B. J. L., Buitelaar, J. K., and van Schie, H. T. (2009). Long-term effects of neurofeedback treatment in autism. Res. Autism Spectr. Disord. 3, 496-501. doi: 10.1016/j.rasd.2008.10.003

Larson, M. J., South, M., Clayson, P. E., and Clawson, A. (2012). Cognitive control and conflict adaptation in youth with high-functioning autism. J. Child Psychol. Psychiatry 53, 440-448. doi: 10.1111/j.1469-7610.2011.02498.x

Llinás, R., and Ribary, U. (1993). Coherent 40-Hz oscillation characterizes dream state in humans. Proc. Natl. Acad. Sci. U S A 90, 2078-2081. doi: 10.1073/pnas. 90.5.2078

Lord, C., Rutter, M., and Le, C. A. (1994). Autism diagnostic interview-revised: a revised version of a diagnostic interview for caregivers of individuals with possible pervasive developmental disorders. J. Autism Dev. Disord. 24, 659-685. doi: $10.1007 / \mathrm{bf} 02172145$

Lorincz, M. L., Kekesi, K. A., Juhasz, G., Crunelli, V., and Hughes, S. W. (2009). Temporal framing of thalamic relay-mode firing by phasic inhibition during the alpha rhythm. Neuron 63, 683-696. doi: 10.1016/j.neuron.2009.08.012

Lovaas, O. I. (1987). Behavioral treatment and normal educational and intellectual functioning in young autistic children. J. Consult. Clin. Psychol. 55, 3-9. doi: 10. 1037//0022-006x.55.1.3

Makeig, S., Westerfield, M., Jung, T. P., Enghoff, S., Townsend, J., Courchesne, E., et al. (2002). Dynamic brain sources of visual evoked responses. Science 295, 690-694. doi: 10.1126/science. 1066168

Maljaars, J., Noens, I., Scholte, E., and van Berckelaer-Onnes, I. (2012). Evaluation of the criterion and convergent validity of the diagnostic interview for social and communication disorders in young and low-functioning children. Autism 16, 487-497. doi: 10.1177/1362361311402857

Martineau, J., Andersson, F., Barthélémy, C., Cottier, J. P., and Destrieux, C. (2010). Atypical activationof the mirror neuron system during perception of hand motion in autism. Brain Res. 1320, 168-175. doi: 10.1016/j.brainres.2010.01.035

McEachin, J. J., Smith, T., and Lovaas, O. I. (1993). Long-term outcome for children with autism who received early intensive behavioral treatment. Am. J. Ment. Retard. 97, 359-372.

Muthukumaraswamy, S. D., and Johnson, B. W. (2004). Changes in rolandic mu rhythm during observation of a precision grip. Psychophysiology 41, 152-156. doi: 10.1046/j.1469-8986.2003.00129.x

Muthukumaraswamy, S. D., Johnson, B. W., and McNair, N. A. (2004). Mu rhythm modulation during observation of an object-directed grasp. Brain Res. Cogn. Brain Res. 19, 195-201. doi: 10.1016/j.cogbrainres.2003.12.001

Neuringer, A. (2002). Operant variability: evidence, functions and theory. Psychon. Bull. Rev. 9, 672-705. doi: 10.3758/bf03196324

Nowlis, D. P., and Kamiya, J. (1970). The control of electroencephalographic alpha rhythms through auditory feedback and the associated mental activity. Psychophysiology 6, 476-484. doi: 10.1111/j.1469-8986.1970.tb01756.x

Oberman, L. M., Hubbard, E. M., McCleery, J. P., Altschuler, E. L., Ramachandran, V. S., and Pineda, J. A. (2005). EEG evidence for mirror neuron dysfunction in autism spectrum disorders. Brain Res. Cogn. Brain Res. 24, 190-198. doi: 10. 1016/j.cogbrainres.2005.01.014

Pantev, C., Makeig, S., Hoke, M., Galambos, R., Hampson, S., and Gallen, C. (1991). Human auditory evoked gamma-band magnetic fields. Proc. Natl. Acad. Sci. U S A 88, 8996-9000. doi: 10.1073/pnas.88.20.8996

Persicke, A., Jackson, M., and Adams, A. N. (2014). Brief report: an evaluation of TAGteach components to decrease toe-walking in a 4-year-old child with autism. J. Autism Dev. Disord. 44, 965-968. doi: 10.1007/s10803-0131934-4

Pfurtscheller, G., Neuper, C., Andrew, C., and Edlinger, G. (1997). Foot and hand area mu rhythms. Int. J. Psychophysiol. 26, 121-135. doi: 10.1016/s01678760(97)00760-5

Pfurtscheller, G., Neuper, C., and Krausz, G. (2000). Functional dissociation of lower and upper frequency mu rhythms in relation to voluntary limb movement. Clin. Neurophysiol. 111, 1873-1879. doi: 10.1016/s1388-2457(00) 00428-4 
Pickett, E., Pullara, O., O'Grady, J., and Gordon, B. (2009). Speech acquisition in older nonverbal individuals with autism: a review of features, methods and prognosis. Cogn. Behav. Neurol. 22, 1-21. doi: 10.1097/wnn.0b013e318190d185

Pineda, J. A. (2005). The functional significance of mu rhythms: translating "seeing" and "hearing" into "doing". Brain Res. Brain Res. Rev. 50, 57-68. doi: 10. 1016/j.brainresrev.2005.04.005

Pineda, J. A., Allison, B. Z., and Vankov, A. (2000). The effects of self-movement, observation and imagination on mu rhythms and readiness potentials (RP's): toward a brain-computer interface (BCI). IEEE Trans. Rehabil. Eng. 8, 219-222. doi: 10.1109/86.847822

Pineda, J. A., Brang, D., Hecht, E., Edwards, L., Carey, S., Bacon, M., et al. (2008). Positive behavioral and electrophysiological changes following neurofeedback training in children with autism. Res. Autism Spectr. Disord. 2, 557-581. doi: 10. 1016/j.rasd.2007.12.003

Pineda, J. A., Juavinett, A., and Datko, M. (2012). Self-regulation of brain oscillations as a treatment for aberrant brain connections in children with autism. Med. Hypotheses 79, 790-798. doi: 10.1016/j.mehy.2012.08.031

Pineda, J. A., Silverman, D. S., Vankov, A., and Hestenes, J. (2003). Learning to control brain rhythms: making a brain-computer interface possible. IEEE Trans. Neural Syst. Rehabil. Eng. 11, 181-184. doi: 10.1109/tnsre.2003.814445

Posthuma, D., and Polderman, T. J. (2013). What have we learned from recent twin studies about the etiology of neurodevelopmental disorders? Curr. Opin. Neurol. 26, 111-121. doi: 10.1097/wco.0b013e32835f19c3

Posthuma, D., de Geus, E. J., Baare, W. F., Hulshoff Pol, H. E., Kahn, R. S., and Boomsma, D. I. (2002). The association between brain volume and intelligence is of genetic origin. Nat. Neurosci. 5, 83-84. doi: 10.1038/nn0202-83

Price, C. J., Ramsden, S., Hope, T. M., Friston, K. J., and Seghier, M. L. (2013). Predicting IQ change from brain structure: a cross-validation study. Dev. Cogn. Neurosci. 5, 172-184. doi: 10.1016/j.dcn.2013.03.001

Rizzolatti, G., and Craighero, L. (2004). The mirror-neuron system. Annu. Rev. Neurosci. 27, 169-192. doi: 10.1146/annurev.neuro.27.070203.144230

Rosenblum, J. (2007). Using TAG to Teach Typing to a Student with Autism. Submitted for TAGteach Level 1 Certification.

Rubenstein, J. L., and Merzenich, M. M. (2003). Model of autism: increased ratio of excitation/inhibition in key neural systems. Genes Brain Behav. 2, 255-267. doi: 10.1034/j.1601-183x.2003.00037.x

Salinas, E., and Sejnowski, T. J. (2001). Correlated neuronal activity and the flow of neural information. Nat. Rev. Neurosci. 2, 539-550. doi: 10.1038/35086012

Salmelin, R., and Hari, R. (1994). Characterization of spontaneous MEG rhythms in healthy adults. Electroencephalogr. Clin. Neurophysiol. 91, 237-248. doi: 10. 1016/0013-4694(94)90187-2

Sayers, B. M., Beagley, H. A., and Henshall, W. R. (1974). The mechansim of auditory evoked EEG responses. Nature 247, 481-483. doi: 10.1038/247481a0

Schoffelen, J. M., Oostenveld, R., and Fries, P. (2005). Neuronal coherence as a mechanism of effective corticospinal interaction. Science 308, 111-113. doi: 10. 1126/science. 1107027

Smith, T., Eikeseth, S., Sallows, G. O., and Graupner, T. D. (2009). Efficacy of applied behavior analysis in autism. J. Pediatr. 155, 151-152. doi: 10.1016/j. jpeds.2009.01.065

Somogyi, E., Kiraly, I., Gergely, G., and Nadel, J. (2013). Understanding goals and intentions in low-functioning autism. Res. Dev. Disabil. 34, 3822-3832. doi: 10. 1016/j.ridd.2013.07.039

Thompson, L., Thompson, M., and Reid, A. (2010a). Functional neuroanatomy and the rationale for using EEG biofeedback for clients with Asperger's syndrome. Appl. Psychophysiol. Biofeedback 35, 39-61. doi: 10.1007/s10484-0099095-0

Thompson, L., Thompson, M., and Reid, A. (2010b). Neurofeedback outcomes in clients with Asperger's syndrome. Appl. Psychophysiol. Biofeedback 35, 63-81. doi: 10.1007/s10484-009-9120-3

Tsiaras, V., Simos, P. G., Rezaie, R., Sheth, B. R., Garyfallidis, E., Castillo, E. M., et al. (2011). Extracting biomarkers of autism from MEG resting-state functional connectivity networks. Comput. Biol. Med. 41, 1166-1177. doi: 10.1016/j. compbiomed.2011.04.004

Ueda, M. (2007). "Implementing TAG to increase compliance in the school environment," in Teaching with Acoustical Guidance (TAG) at Applied Behavior Consultants Inc. Symposium Conducted at the 25th Annual Western Regional Conference, eds J. Morrow and J. Vargas (Burlingame, CA: California Association for Behavior Analysis).

van den Bos, W., Crone, E. A., and Güroğlu, B. (2012). Brain function during probabilistic learning in relation to IQ and level of education. Dev. Cogn. Neurosci. 2(Suppl. 1), S78-S89. doi: 10.1016/j.dcn.2011.09.007

Villalobos, M. E., Mizuno, A., Dahl, B. C., Kemmotsu, N., and Muller, R. A. (2005). Reduced functional connectivity between V1 and inferior frontal cortex associated with visuomotor performance in autism. Neuroimage 25, 916-925. doi: 10.1016/j.neuroimage.2004.12.022

Wainer, A. L., and Ingersoll, B. R. (2013). Disseminating ASD interventions: a pilot study of a distance learning program for parents and professionals. J. Autism Dev. Disord. 43, 11-24. doi: 10.1007/s10803-012-1538-4

Whittington, M. A., Traub, R. D., Faulkner, H. J., Stanford, I. M., and Jefferys, J. G. (1997). Recurrent excitatory postsynaptic potentials induced by synchronized fast cortical oscillations. Proc. Natl. Acad. Sci. U S A 94, 12198-12203. doi: 10. 1073/pnas.94.22.12198

Williams, J. H., Waiter, G. D., Gilchrist, A., Perrett, D. I., Murray, A. D., and Whiten, A. (2006). Neural mechanisms of imitation and 'mirror neuron' functioning in autistic spectrum disorder. Neuropsychologia 44, 610-621. doi: 10.1016/j. neuropsychologia.2005.06.010

Williams, J. H., Whiten, A., Suddendorf, T., and Perrett, D. I. (2001). Imitation, mirror neurons and autism. Neurosci. Biobehav. Rev. 25, 287-295. doi: 10 1016/s0149-7634(01)00014-8

Winkle, M. (2007). TAGteach as an Occupational Therapy Intervention Strategy. Available online at: http://www.tagteach.com/Resources/Documents/Melissa _Winkle_OT.pdf

Conflict of Interest Statement: The authors declare that the research was conducted in the absence of any commercial or financial relationships that could be construed as a potential conflict of interest.

Received: 23 February 2014; accepted: 06 July 2014; published online: 07 August 2014. Citation: Pineda JA, Friedrich EVC and LaMarca K (2014) Neurorehabilitation of social dysfunctions: a model-based neurofeedback approach for low and highfunctioning autism. Front. Neuroeng. 7:29. doi: 10.3389/fneng.2014.00029 This article was submitted to the journal Frontiers in Neuroengineering. Copyright (C) 2014 Pineda, Friedrich and LaMarca. This is an open-access article distributed under the terms of the Creative Commons Attribution License (CC BY). The use, distribution or reproduction in other forums is permitted, provided the original author(s) or licensor are credited and that the original publication in this journal is cited, in accordance with accepted academic practice. No use, distribution or reproduction is permitted which does not comply with these terms. 